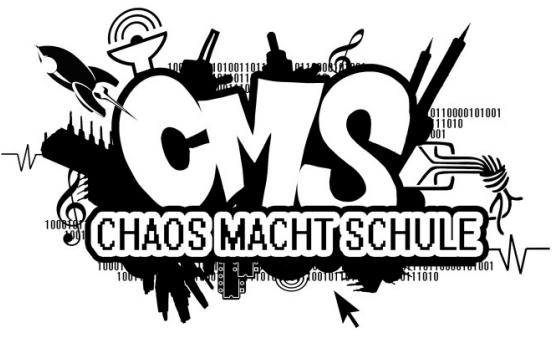

\section{Chaos macht Schule}

\section{BORIS KRAUT}

Piraten, Hacker, Kriminelle. Das Bild von Hackern, das viele Medien transportieren, ist häufig negativ besetzt und prägt die öffentliche Wahrnehmung maßgeblich. Selbst wer schon mal etwas vom Chaos Computer Club (CCC) gehört hat, dürfte ihn eher mit den Begriffen Technik oder immer häufiger auch Gesellschaft und Politik in Verbindung bringen. Doch Schule und Bildung? Was auf den ersten Blick erstaunlich wirkt, macht durchaus Sinn: Zum einen war der freie Austausch von Informationen, das Verständnis von Technik und Welt und die Weitergabe von Wissen schon immer ein Teil der Hackerkultur, zum anderen ist Bildung ein Bereich, der gesellschaftspolitischer nicht sein könnte. Hier werden die Weichen für das zukünftige Zusammenleben und Werteverständnis gelegt. In einer Welt, die immer mehr von Technik durchdrungen ist, ist es daher auch Aufgabe der Schulen, die kommenden Generationen darauf vorzubereiten - Stichwort „Medienkompetenz". Da das Thema jedoch im letzten Jahrzehnt sträflich vernachlässigt wurde, gibt es schon seit 2007 Aktivisten im CCC, die Schülern, Lehrern und Eltern bei diesem Themenbereich hilfreich zur Seite stehen: Chaos macht Schule (CmS).

\footnotetext{
Wie der Club in die Schulen kam ...

Der Chaos Computer Club lebt Dezentralität. Anstatt einer von oben vorgegebenen Agenda zu folgen, beginnen viele Projekte privat bei einzelnen Mitgliedern und werden dann in den jeweiligen „eigenständigen CCC-Ortsgruppen“, den so genannten Erfahrungsaustauschkreisen (Erfa), und ggf. über Erfa-Grenzen hinweg bekannt gemacht und diskutiert.
}

Neben Angeboten für Jugendliche im Allgemeinen (vgl. „u23“-Projekt) begannen einzelne Erfas ab 2007 direkt an die Schulen zu gehen - teils aus eigenem Antrieb, doch meist auf Anfrage. Auslöser war die Einsicht, dass im Bildungsbereich etwas schief läuft: Die inzwischen nicht mehr ganz so „,neuen“ Medien wurden ,,alltäglich“, aber viel zu oft wurden die Schüler damit allein gelassen.

Während Eltern die Verantwortung an die Schulen gaben, waren viele Lehrer nicht für diese Aufgabe vorbereitet. Die im Bildungsplan 2004 erwähnte Medienkompetenz als Querschnittsaufgabe war zwar von der Intention her richtig - der mediale Umwälzungsprozess macht vor keinem Unterrichtsfach halt - verleitete jedoch dazu, der Aufgabe nicht die nötige Zeit einzuräumen oder sie ganz zu streichen: Man muss schließlich mit dem Lehrstoff durchkommen, „digitale Medien“ soll da lieber der Informatik-Lehrer unterrichten! Und auch die Politik reagierte eher träge auf den Missstand, sodass man in vielen Erfas die unterschiedlichsten Schulprogramme auflegte: Vorträge, Workshops, spezielle Elternabende, Hardware-Basteleien mit Schülern oder gar die Ausbildung von Medienscouts in Kooperation mit Stadt, Polizei und Jugendund Sozialeinrichtungen.

\section{Digital Natives}

Insgeheim haben wohl alle gehofft, dass mit der Ankunft der „digital natives“ die Sache für Schüler wie für Lehrer von allein regeln würde - ein Fehler. Wie es sich heraus stellte, konnten nachkommende Generationen zwar häufig besser mit der Technik umgehen, sie zumindest bedienen, doch eine wirkliche tiefgreifende und vollumfängliche Medienkompetenz prägte sich nicht „einfach so“ aus: Sie muss erlernt werden!

Daraus ergeben sich weitreichende Konsequenzen:

(1) Lernen findet natürlich auch außerschulisch statt, aber bei einer so tiefgehenden Veränderung der Lebenswelt ist es Aufgabe der Schule, darauf vorzubereiten. Zwar können außerschulische Initiativen wie $\mathrm{CmS}$ kurzfristig einspringen und ausgleichend wirken, eine dauerhafte Lösung sind sie nicht. Auch wenn das Bild von rein tech- 
nisch geprägten Hackern eher ein Klischee ist - im CCC finden sich neben Informatikern, Künstlern, Bastlern, politischen Aktivisten und weiteren auch Lehrer und Betreuer - so ist klar, dass nur ein kleiner Teil von uns tiefere pädagogische Kenntnisse mitbringt. Genauso umfasst Medienkompetenz deutlich mehr als die von uns gesondert herausgegriffenen digitalen Inhalte. Wir leisten gerne unseren bescheidenen Beitrag, aber auf lange Sicht müssen Pädagogen diese Arbeit übernehmen: Es ist und bleibt die Aufgabe von Schule!

Sie muss Schülern weiterhin, also auch für „,neue Medien“, das Handwerkszeug für eine kritische Mediennutzung bieten. Dazu bedarf es einer anregenden Lernumgebung und kompetenten Lehrern. Für eine solche Lernumgebung muss die passende Ausstattung der Schulen und auch die der Schüler gewährleistet werden: Persönliche Geräte sind wichtig, da sich ein alltäglicher Umgang nicht an für spezielle Fächer zu bestimmten Zeiten freigegebenen Computern erlernen lässt, sondern eben nur, wenn er im Alltag gelebt wird. Dazu gehört die private Nutzung ebenso wie die fachbezogene Nutzung, die trotz der ständigen Verfügbarkeit nur gezielt stattfinden sollte - auch damit müssen Schüler umgehen lernen. Die Umsetzung einer solchen Forderung ist in Zeiten stets klammer Kassen strittig. Es muss aber sichergestellt werden, dass finanzschwache Schüler nicht benachteiligt werden. Eine Komplettausstattung durch die Schulen wird kaum machbar sein, aber eine Bezuschussung bestimmter Geräte ist denkbar: Minicomputer wie der Raspberry-Pi sind eine gute Idee und laden zum Basteln ein, als Alltagsgerät sind sie jedoch weniger brauchbar. Zum Glück gibt es inzwischen auch genügend erschwingliche Laptops und Tablets. Dabei gilt es, eine langfristige Bindung an einen Hard- bzw. Softwarehersteller (VendorLock-In), wie bei speziellen iPad-Klassen, zu vermeiden (s. „Werkzeugbegriff"). Hier sei auch noch auf ein weiteres Problemfeld hingewiesen: Dass Schüler ihre eigenen Geräte mitbringen, scheint wünschenswert, auf Seiten der Lehrer sollte man darauf achten, dass Bring-Your-Own-Device (BYOD) auch datenschutzrechtliche Fragen aufwirft: Sind die privaten Daten der Schüler ausreichend geschützt? Werden die Bestimmung z.B. der „Verwaltungsvorschrift zum Datenschutz an öffentlichen Schulen“ (Az.: 11-0551.0/38) eingehalten?

Wichtiger als die Technik ist aber das Schaffen von verpflichtenden Zeitkontingenten: Hier wird Fundamentales für die Zukunft, für das Leben gelernt. Dieses Wissen darf nicht davon abhängig gemacht werden, ob man neben dem normalen „Stoff“ noch Zeit dafür findet oder ob der Lehrer das Thema behandeln will. Wie diese Zeit verteilt wird, ist zu diskutieren: Die Vermittlung von Medienkompetenz als reine Querschnittsaufgabe verschiedener Fächer stößt einerseits an das Zeitproblem - man hängt ja immer dem Plan hinterher -, andererseits wird sie der Wichtigkeit des Themas nicht gerecht. Sie über ein eigenes Fach im Sinne von ,informationstechnischer Grundbildung“ (ITG) oder dem Fach „Medienkunde“ (Thüringen) zu thematisieren, ist ein anderer Weg. Dadurch lässt sich inhaltlich viel abdecken, doch eine wirkliche „Alltagskompetenz" wird sich durch ein paar Stunden Medienbildung nicht ausbilden. Es braucht eine umfangreiche Beschäftigung mit der Thematik in beidem einem gesonderten Unterricht, wie auch in den anderen Fächern - sowie natürlich in der Freizeit.

Auch die Lehrer brauchen ein attraktives Weiterbildungsangebot - und zwar nicht nur für ältere Lehrergenerationen: Wie bei den Schülern hat sich auch bei den Lehrern die Hoffnung auf „digital natives“ nicht erfüllt. Selbst aktuelle Referendare sind nicht per se viel kompetenter im Umgang mit digitalen Medien. In letzter Konsequenz muss schon im Lehramtsstudium verpflichtend das Thema behandelt werden. Dabei bleiben diese Forderungen, wie sie auch in der aktuellen baden-württembergischen Prüfungsordnung für die Werk-, Haupt- und Realschulen (WHRPO I 2011) aufgeführt sind, Lippenbekenntnisse, solange sie nicht flächendeckend und mit entsprechender Ernsthaftigkeit umgesetzt werden. In naher Zukunft werden solche Inhalte einen ähnlichen Stellenwert einnehmen wie das Sprechen, das vielerorts mit der gesonderten Lehrveranstaltung „Grundlagen des Sprechens“ gewürdigt wird. Es wäre zu begrüßen, wenn analog eine verbindliche Veranstaltung „Grundlagen der Medienbildung" an allen Hochschulen in Baden-Württemberg angeboten werden könnte, wie dies bereits seit einigen Semestern an der PH Ludwigsburg mit der „Einführung in die Medienpädagogik“ (2 SWS) der Fall ist. Im Rahmen von „Chaos macht Schule" unterstützten wir schon an mehreren Pädagogischen Hochschulen und Universitäten die Lehrerbildung und wollen diese Arbeit auch in Zukunft weiter intensivieren.

- Der Umgang mit Medien muss gelernt werden.

- Es ist Aufgabe von Schule, für eine angemessene Grundbildung zu sorgen.

- Dazu gehört eine verbesserte Ausstattung der Schulen und der Schüler. 
- Medienbildung muss in den Bildungsplänen inhaltlich und zeitlich tiefer verankert werden.

- Inhalte müssen dabei universell formuliert sein und genügend Raum zur Reaktion auf die schnelllebige IT-Welt lassen.

- Lehrer müssen an entsprechenden Fortbildungen teilnehmen.

- Schaffung von verpflichtenden Veranstaltungen „Grundlagen der Medienbildung“ in der Lehrerbildung sowie von vertiefenden Angeboten zur Medienbildung.

(2) Tiefgreifend und vollumfänglich kann Medienbildung nur dann sein, wenn sie nicht bei Buch, Zeitung und Fernsehen stehenbleibt: „Neue Medien“ dürfen nicht ausgeklammert oder nur oberflächlich behandelt werden! Rein auf Computer und Internet ausgerichtet ist Medienbildung im Umkehrschluss aber ebenfalls entartet und kontraproduktiv.

Greift man bei der Aufgliederung des Begriffs Medienkompetenz (vgl. Baacke) auf die bekannten Felder Medienkritik, -kunde, -nutzung und -gestaltung zurück, ergibt sich folgendes Bild:

- Medienkritik: Die derzeitige Medienbildung mag bei traditionellen Medien noch funktionieren, versagt jedoch meist bei den „neuen“. Weder das Weglassen bzw. thematisch Kleinhalten noch das überschwängliche Anpreisen führt zu einer wirklich kritischen Nutzung. Tatsächlich ist bei vielen Jugendlichen wie auch bei den meisten Erwachsenen kaum jemand zu finden, der wirklich begründete Entscheidungen für oder gegen einzelne Dienste fällt: Wikipedia ist nicht zitierfähig und Facebook nutzt man, weil dort alle sind. Kaum jemand fragt sich, was er wirklich tun möchte und was dafür die geeigneten Medien, Dienste oder Programme sind. Und selbst bei einer genauen Abwägung von Vor- und Nachteilen wird meist sehr egoistisch argumentiert; eine gesellschaftliche Dimension fehlt meist: Zwar mag es für mich ideal sein, etwas zu nutzen, aber die Nutzung wird weitreichende Folgen für andere haben (s. „Werkzeugbegriff").

- Medienkunde: Während Schüler bei den bisherigen Medien meist immer noch ein solides Basiswissen erlangen, werden beispielsweise beim Themenkomplex Internet nur einzelne Dienste besprochen. Exemplarische Inhalte sind notwendig, sie müssen aber grundlegende Konzepte veranschaulichen und dürfen nicht den Blick auf die Vielfalt der neuen Möglichkeiten nehmen. Das Internet ist nicht nur das Web. Und auch das Web besteht nicht nur aus Wikipedia, Facebook und Google.
- Mediennutzung: Hier bestätigen sich die meisten Hoffnungen, denn vielen „digital natives" fällt es leicht, sich sowohl in den alten als auch den neuen Medien zu bewegen. Die reine Anwendung ist kaum ein Problem, sie erlernen spielerisch und selbstgesteuert sowohl den rezeptiven Umgang mit den Medien als auch die Interaktion mit diesen - und anderen Nutzern. Leider herrscht auch hier ein sehr eingeengter Blick auf die digitalen Medien vor. Was Bedienung und Funktionsumgang angeht, sind Erfahrungen hier nicht immer übertragbar.

- Mediengestaltung: Innovativer und kreativer Umgang mit Medien wird in Schulen nur teilweise geübt. Wieder sind die ,alten“ Medien führend, wobei man auch hier fragen kann, wie viel Kreativität sich in den teils recht starren Aufgabenstellungen entfalten lässt. Auch „neue“ Medien, allen voran Audio- oder Videoproduktionen, sind immer wieder anzutreffen. Die Nutzung des Internets beschränkt sich - falls überhaupt - auf Recherche, Blogs oder Schulwikis. Gerade was die kooperative Gestaltung und Entwicklung angeht wird zu wenig geboten. Ein Vorwurf, der auch einige CmS-Veranstaltungen trifft: Schulzeit ist kostbar und meistens wird man aufgrund von ,aktuellen Problemen“ (Cybermobbing, Stalking, Medienkonsum usw.) eingeladen. Weniger warnende und dafür mehr aktiv erforschende Veranstaltungen wären begrüßenswert.

Medienkompetenz ist dabei nicht nur Selbstzweck oder eine rein persönliche Entscheidung, sondern besitzt immer auch eine gesellschaftliche Dimension: Unsachgemäße Mediennutzung kann nicht nur für den Betroffenen, sondern auch für andere - man denke an von Spammern gekaperte Privat-PCs — oder die Gesellschaft im Ganzen - z.B. bei politischen Entscheidungen - haben. Zudem kann es in einer medial und technisch geprägten Gesellschaft keine mündige Teilhabe ohne Medienkompetenz geben. Umfassende und nachhaltige Medienkompetenz darf kein Elitenwissen sein.

\section{Medienkompetenz und „digitale Mündigkeit“}

Förderung der Medienkompetenz ist für Schulen keine neue Aufgabe, doch auf das Aufkommen der digitalen Medien wurde vielerorts nicht reagiert. Im besten Fall wurden diese „neuen“ Medien gleichberechtigt neben den anderen behandelt, meist jedoch eher untergeordnet oder gar nicht. Doch während sie natürlich nur ein Teilgebiet darstellen, so sind sie von fundamentalerer Bedeutung, denn sie sind viel mehr als nur ein weiteres Medium: 
Die Digitalisierung nimmt fast alle bisherigen Kommunikationsformen und Medien in sich auf und macht sie über ein Metamedium (vgl. McLuhan) bzw. Hypermedium (vgl. Winkler u.a.) nutzbar, das wir heute beispielsweise als Internet kennen. Man könnte also argumentieren, dass ein Text immer ein Text bleibt, ganz egal, ob er gesprochen, in Stein gemeiBelt, auf Papier geschrieben oder in einem Blog veröffentlicht wird. Doch ist diese Reduktion auf den Inhalt bzw. sogar nur auf den Text unangebracht. Wer den Informationsträger Stein nutzt, wird seine Worte mit Bedacht wählen, während das Internet die Kommunikation alltäglich, einfach und schnell macht. Die Möglichkeit, unüberlegte und teils banale Texte zu verfassen, führt aber weder zwangsläufig dazu noch ist sie per se negativ: Wer hat nicht schon mal einen guten Gedanken schnell fixieren müssen, ohne dass die Zeit blieb, ihn in schöne Worte zu packen? Wer war nicht schon mal froh darum, sich nicht immer nur mit den schwersten Problemen der Menschheit auseinandersetzen zu müssen, und hat Entspannung und Sinn im scheinbar Banalen gefunden? Smalltalk ist kein sinnloses Gerede, es kann durchaus entspannend sein und hilft beim Aufbau von Kontakten. Das Wechseln des Informationsträgers ist also deutlich mehr, als man anfangs vermuten mag. Es würde daher auch niemand dem Übergang von mündlicher $\mathrm{zu}$ schriftlicher Überlieferung oder der Erfindung des Buchdrucks unterstellen, dass sich damit nur der Träger oder die Technik geändert hat. Und so gab es schon die ersten Überlegungen, dass hier neben Lesen, Schreiben und Rechnen eine neue Kulturtechnik entsteht. Während man sich prinzipiell darauf verständigen konnte, dass hier zwar lediglich die alten in veränderter Form aufgegriffen werden (vgl. Liessmann), scheint in einem Medium, das es für jeden so leicht macht, Daten zu erzeugen, eine weitere Fähigkeit massiv an Bedeutung $\mathrm{zu}$ gewinnen: Informationsfindung, -bewertung und -prüfung.

Es scheint jedoch klar, dass man es hier nicht nur mit einem massiven Geschwindigkeitszuwachs und einer besseren Maschinenlesbarkeit zu tun hat, sondern dass aufgrund der gefallenen Kosten ein Massenmedium prinzipiell für alle gleichberechtigt zur Verfügung steht. Man ist nicht mehr nur Empfänger von Nachrichten, die finanzstarke Unternehmen produziert und für wichtig befunden haben, man kann selbst Sender werden!

Dieses Aufbrechen von Hierarchien verläuft nicht problemlos: Akteure, die früher Wissen und Kommunikation allein kontrollieren konnten, verlieren teilweise an Einfluss und Autorität, werden angezweifelt, korrigiert, belehrt. Eine Vorstellung, die auch einigen
Lehrern unangenehm sein könnte. Doch auch der Umgang mit der gewonnen Freiheit und Macht muss erlernt werden: Dass das Internet natürlich kein rechtsfreier Raum ist - es gelten dieselben Gesetze, auch wenn diese an ihre nationalen Grenzen gebunden sind - und dass man hier häufiger unwissentlich mit einer weltweiten Öffentlichkeit kommuniziert, wo man doch nur privat zu einer Feier einladen wollte, muss erst nachvollzogen und begriffen werden. Wir erleben hier eine Potenzierung der Effekte - im Positiven wie auch im Negativen. Wir benötigen einen verantwortungsvollen und sozialverträglichen Umgang mit Technik: Digitale Mündigkeit.

Und genau hier können Lehrer schon viel beitragen, denn es lässt sich bisheriges Wissen über das Zusammenleben in einer Gesellschaft leicht übertragen, wenn man ein grundlegendes Verständnis der Technik hat - ohne dieses geht es jedoch nicht.

\section{Implikationen des Werkzeugbe- griffs}

So wird z.B. Facebook nicht nur für persönliche Zwecke, sondern auch immer häufiger als „Tool“ für den Unterricht genutzt. Ist das wirklich überdacht? Der Begriff ist aus zweierlei Hinsicht problematisch: Zum einen bedeutet elektronische Kommunikation heutzutage viel mehr, sie ist nicht nur reines Werkzeug, sondern steht - ähnlich wie „Auto“ nicht nur für „Fahrzeug“, sondern für „Mobilität“ - für deutlich mehr - sowohl bei privater Nutzung als auch im schulischen Einsatz. Zum anderen ist der Begriff verharmlosend und verbirgt Komplexität: Ein Werkzeug tut nur eine Sache, ist kontrollierbar und sein Verhalten wiederholbar. Die meisten Onlinedienste sind das nicht. Sie haben AGB, denen ggf. auch die Schüler bzw. Eltern zustimmen müssen. Sie mögen vermeintlich kostenfrei sein, müssen sich aber finanzieren. Wie passiert das und welches Interesse verfolgt das Unternehmen? Was geschieht anderenfalls mit meinen Daten und Dateien? Kann ich bei Bedarf einfach wechseln und diese mitnehmen, sie also ohne Verlust exportieren und importieren? Wir alle sollten uns nicht zu sehr über die neue Wunderwelt des Web 2.0 freuen und uns insbesondere nicht zu sehr von ihr abhängig machen - gerade im Bereich der Bildung!

So wie man nicht jedes im Netz gefundene Bild automatisch auch für eigene Zwecke nutzen darf, muss sich auch langsam die Erkenntnis ausprägen, dass „kostenlos“ nicht gleichbedeutend mit „frei“ ist. Einen grundlegenden Einblick geben hier die vier Softwarefreiheiten (vgl. FSF); sie beinhalten: 
1. Das Programm zu jedem Zweck auszuführen.

2. Das Programm zu untersuchen und $\mathrm{zu}$ verändern.

3. Das Programm zu verbreiten.

4. Das Programm zu verbessern und diese Verbesserungen zu verbreiten, um damit einen Nutzen für die Gemeinschaft zu erzeugen.

Da diese Grundsätze deutlich besser in den Bildungsbereich passen als die Bedingungen proprietärer Software, sollten Schulen vermehrt auf freie Software setzen. Während für die Schulen nicht das „kostenlos“-Argument im Vordergrund stehen sollte - auch freie Software kann durchaus Kosten verursachen, z.B. Wartung - ist dies für die Schüler wirklich wichtig. Sie müssen auch zuhause die Möglichkeit auf Nutzung haben, da nur durch den alltäglichen Umgang die benötigten Kompetenzen ausgebildet werden.

Diese dürfen allerdings auch bei freier Software nicht im Auswendiglernen von „Klickanleitungen“ liegen. Es müssen stattdessen Vielfalt und generelle Konzepte gelehrt werden, die befähigen, sich in der schnelllebigen Softwarewelt dauerhaft zurechtzufinden - egal ob sich z.B. Änderungen an der Bürosuite aufgrund einer neuen Version ergeben oder weil das komplette Produkt gewechselt wird. Transferleistungen werden auch hier immer wichtiger.

Doch auch die Hardware muss mit Bedacht gewählt werden. Es muss verhindert werden, dass man in dem Willen, „modernen“ Unterricht vorzuleben, sich z.B. allein auf eine geschlossene Hard- und Softwareplattform einlässt (vgl. iPad-Klassen; Vendor-Lock-In) auch nicht, wenn die enormen Anschaffungskosten z.B. durch Spenden gedeckt werden. Vielmehr sollte hier die Vielfalt auch als Chance begriffen werden: Neben den von Schulen beschafften Geräten, die nicht geschlossen sein sollten, können entsprechende Geräte der Schüler ebenfalls genutzt werden. Zwar muss klar sein, wann Smartphones erlaubt sind und wann nicht, aber anstatt nur Verbote zu erlassen, sollten sich Lehrer überlegen, wie die Geräte sinnvoll in den Unterricht zu integrieren sind. Sie sollten Schülern zeigen, welche Möglichkeiten sich hier bieten, anstatt sie in die heimliche FacebookNutzung unter der Schulbank zu treiben.

Das führt unweigerlich $\mathrm{zu}$ der Forderung nach plattformübergreifenden und digitalen Lerninhalten. Leider sind solche Angebote seitens der etablierten Schulbuchverlage kaum vorhanden und häufig auf einzelne Systeme beschränkt und oder mit „Digital Rights Management“ (DRM) belastet. Hier könnten freie Lehrmittel und Inhalte, „Open Educational Resources" (OER), eine Option sein.

\section{Zusammenfassung}

Der Chaos Computer Club versucht schon immer, auch Jugendliche für einen spielerischen, forschenden Umgang mit Technik zu begeistern, so dass sie Nutzen und Gefahren kennen und beurteilen lernen. Nur so werden sie auch im Digitalen zu einem selbstbestimmten Individuum und mündigen Teil der Gesellschaft. Sie lernen Technik aktiv und gezielt zu nutzen, werden also nicht mehr von ihr kontrolliert und getrieben.

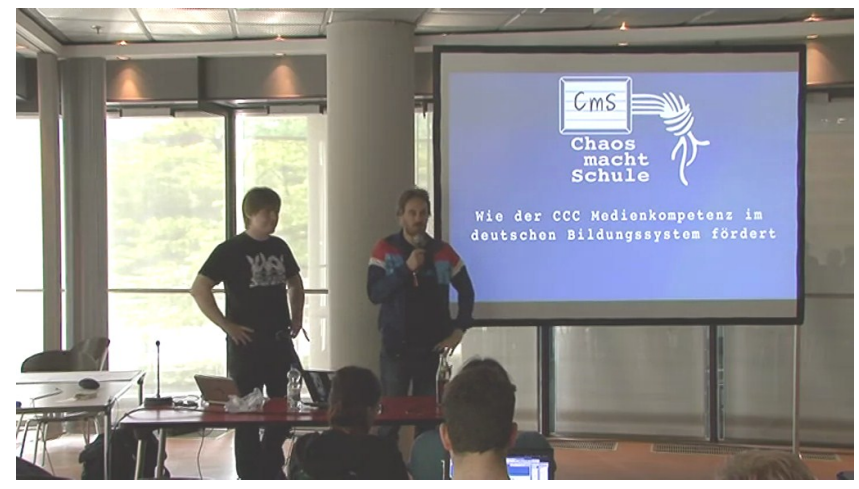

Da Schulen und Lehrer oftmals mit der Situation überfordert sind, versuchen wir vermehrt direkt in Unterrichtsstunden zu gehen. Eine flächendeckende und dauerhafte Abdeckung ist aber rein mit ehrenamtlichen Helfern nicht zu erreichen: Weder haben wir dazu die personellen Mittel, noch sind wir als Techniker die erste Wahl. Wir suchen daher explizit den Kontakt zu Pädagogen, um gemeinsam an diesem wichtigen Ziel zu arbeiten.

Die Politik muss die Weichen für ein erneuertes Bildungswesen stellen, das den Herausforderungen der modernen Zeit gewachsen ist. Medienkompetenz muss in den Bildungsplänen so gestärkt werden, dass diese Querschnittsaufgabe ernst genommen wird und werden kann. Dazu muss Lehrern und Schülern die nötige Zeit genauso zur Verfügung stehen wie auch die technische Ausstattung. Entsprechende Lernmaterialien müssen so angepasst werden, dass sie grundlegende Fähigkeiten vermitteln und nicht nur schnell vergängliche Anleitungen liefern. Auf Seiten der Lehrerbildung müssen nicht nur Fortbildungen angeboten werden, sondern auch die Studien- und Prüfungsordnungen entsprechende Änderungen erfahren: Lehramtsstudierende sollen passende Veranstaltungen nicht nur zusätzlich, sondern verpflichtend belegen. 


\section{Links}

Information und Kontakt (bundesweit)

- https://ccc.de/schule

- https://ccc.de/de/updates/2012/ lernplan-statt-lehrplan

- mailto:schule@ccc.de

Dokumentation (exemplarisch)

- Ulm: https://www.ulm.ccc.de/ ChaosMachtSchule

- Mannheim: https://www.ccc-mannheim.de/wiki/ Chaos_macht_Schule

- Karlsruhe: https://entropia.de/CMS

- Hamburg: https://cms.hamburg.ccc.de/

- U23-Projekt Köln: https://koeln.ccc.de/u23/

- Vortrag PH Ludwigsburg: https://entropia.de/PHLB-Kolloquium
Boris Kraut ist Student an der PH Karlsruhe und engagiert sich ehrenamtlich im $\mathrm{CmS}$ Projekt des CCC. Neben Vorträgen und Workshops für Schüler, Lehrer und Eltern versucht er seit einiger Zeit auch die Medienbildung in den Lehramtsstudiengängen voranzutreiben.

Am 21. Mai 2012 war er auf Einladung von Prof. Dr. Horst Niesyto Gastreferent im „Medienpädagogischen Kolloquium“ an der PH Ludwigsburg, um dort das Bildungsangebot des CCC vorzustellen. Dieser Artikel gibt einen kleinen Einblick in die Beweggründe und Ausrichtung von $\mathrm{CmS}$. Eine längere Version, die auch inhaltliche Beispiele zu sozialen Netzwerken und Urheberrecht beinhaltet, sowie die komplette Dokumentation des Vortrags sind im Wiki des CCC Karlsruhe verfügbar. 